Check for updates

Cite this: Mater. Adv., 2022, 3,282

Received 10th September 2021 Accepted 7th October 2021

DOI: $10.1039 / \mathrm{d} 1 \mathrm{ma} 00839 \mathrm{k}$

rsc.li/materials-advances

\section{Single wavelength colour tuning of spiropyran and dithienylethene based photochromic coatings $\dagger$}

\author{
Ruben Feringa, (D) Harmke S. Siebe, (D) W. J. Niels Klement, (D) Jorn D. Steen (D) \\ and Wesley R. Browne (D) *
}

\begin{abstract}
Controlling the transmission of thin films with external stimuli is an important goal in functional optical materials and devices. Tuning is especially challenging where both broad band (neutral density filtering) and spectrally varied (colour) transmission are required. The external control provided by photochemically driven switching, between transmission levels and colours, is functionally simple from a device perspective. The limits due to the spectral ranges of individual photochromic compounds can be overcome by combining several photochromes within one material or device. Here we show that a combination of photochromic molecular switches immobilised in a PMMA polymer matrix enables tuning of colour and transparency. We show that only a single excitation wavelength is required through the use of the primary inner filter effect and the layered construction of the films in which the photochromes nitrospiropyran (NSP), and nitrothiospiropyran (TSP) or 1,2-bis-terthienylhexafluorocyclopentene (DTE) are separated spatially. The approach taken circumvents the need to match photochemical quantum yields and thermal reactivity of the component photochromes. The photochemical switching of the films was characterised by UV/vis absorption spectroscopy and shows that switching rates and photostationary states are limited by inner filter effects rather than the intrinsic properties of photochromes, such as photochemical quantum yields and thermal stability. The photochemical behaviour and stability of the photochromes in solution and in the PMMA films were compared and the concentration range over which self-inhibition of photochemical switching occurs was established. The rate of photochemical switching and the difference in transmission between the spiropyran and merocyanine forms in solution enable prediction of the performance in the films and enable rational design of colour tuning ranges and responsivity in thin film filters.
\end{abstract}

Chromic materials are ubiquitous in nature and nano-materials provide a wide range of functions, such as display, camouflage, and thermal regulation, with colour provided either through structure, e.g., by optical interference such as in the iridescent colours of butterfly and beetle wings, ${ }^{1,2}$ or from absorption or emission of light in the visible spectrum. ${ }^{3}$ The application of coloured materials ranges from decoration and art to optical filters. In situ control of colour can be achieved using external stimuli, such as pressure, electric fields or light, both in nature ${ }^{4}$ and in synthetic materials. ${ }^{5}$ Examples of which are tuneable optical filters based on liquid crystals ${ }^{6}$ or acousto-optical filters (AOTFs), ${ }^{7}$ in which optical transmission is controlled by

Molecular Inorganic Chemistry, Stratingh Institute for Chemistry, Faculty of Science and Engineering, University of Groningen, Nijenborgh 4, 9747AG Groningen,

The Netherlands. E-mail: w.r.browne@rug.nl

$\dagger$ Electronic supplementary information (ESI) available: Details of synthesis and characterisation of TSP and additional spectral data. See DOI: 10.1039/d1ma00839k changes in microstructure using electric fields or sound. AOTFs are used for medical applications ${ }^{8}$ and in astronomy. ${ }^{9}$

Photochromic materials based on compounds that can change their structure and their visible absorption with light as an external stimulus, provide a wide range of variations in responsivity and colour. Synthetic tuning of known photochromes enables fine-tuning of colour, thermal stability and the excitation wavelengths required..$^{10,11}$ The efficiency of the photochemical reactions involved in molecular photochromism and the colours obtained depend on the quantum yield of the photochromic process in each direction, as well as the electronic absorption spectra of both states of the photochrome. The high molar absorptivities of common photochromes mean that they are well-suited for incorporation as dilute components in solid supports to form photochromic materials, which find applications as diverse as organic transistors and sunglasses, ${ }^{12-14}$ as well as recent advances in colour changing films. ${ }^{15,16}$ Barachevsky et al. recently reported a 
multifunctional layered film for control of illumination together with thermal management of indoor spaces provided by windows. ${ }^{17}$ The effect of irradiation with broad band ambient light depended on the side of the multilayer glass irradiated due to the inclusion of one layer that permanently reflects nearinfrared light and a second photochromic layer.

Molecular systems typically absorb significantly in only part of the visible spectrum, but tuning of colour over an entire range can be achieved using multi-photochromic systems, in which two photochromic compounds are combined either with covalent tethering or as simple mixtures. ${ }^{13,18}$ Covalent connection of different photochromes has the advantage that the ratio of photochromes is controlled precisely and colour tuning is more predictable. A disadvantage of this approach is synthetically demanding and requires careful matching of photochemical and electronic properties. Colour tuning can be achieved with so-called orthogonal multiphotochromes, in which combinations of photochromes are chosen such that their spectral overlap allows for selective excitation of one photochrome or the other depending on the wavelength of light used. ${ }^{19}$

Combining photochromes in a single material by physical mixing is simpler and allows for more rapid optimisation, although it requires that the photochromes are chemically similar to ensure a homogenous distribution through the film. Using two differently coloured photochromes that can be switched using the same wavelength of light simplifies operation. However, with mixtures of photochromes, the intermediate transmission depends on the properties of each component, while the final (at the photostationary state, PSS) transmission spectrum depends only on the ratio of photochromes present. Intermediate transmission spectra can be obtained from the mixture to some extent by choosing components with appropriate ratios of photochemical quantum yields, such that one component reaches its PSS faster than the other. Since the photochemical quantum yields of photochromes cannot yet be predictably tuned by changes in the chemical structure, ${ }^{20}$ this approach is relatively limited. Furthermore, when the photochromes are connected covalently, or present at high concentrations in the films, the effect of aggregation and energy transfer on the photophysical properties needs to be taken into account also in addition to bimolecular degradation pathways. ${ }^{21}$

Here we show that the optical transmission/colour of a photochromic material can be tuned with a single wavelength of excitation. Our approach is to take advantage of the primary inner filter effect and the physical separation of the photochromes in stacked layers within the host matrix. Layers of photochrome-containing poly-methyl methacrylate (PMMA) are deposited by sequential spin-coating, using intermediate layers of hydroxyethyl cellulose (HEC) that prevent dissolution of prior PMMA layers in the solvent used for spin-coating the subsequent layer of PMMA. The use of two different polymers for spin-coating multiple layers was reported previously for multilayered high reflection mirrors. ${ }^{22}$ This approach allows for control of the position of the photochromes (in depth) and overall composition of the polymer film formed. Spiropyran photochromes (nitrospiropyran (NSP), nitrothiospiropyran (TSP), Scheme 1) are selected as their absorption in the UV region does not change significantly during photochromic switching. This property is important in the material. Each layer of the material provides colour upon photochemical switching to the open (merocyanine) form and also prevents photoexcitation of the photochromes present in subsequent layers due to the primary inner filter effect. The wavelength dependence of the transmission of the material is controlled in a predictable manner by the side from which the multi-layered
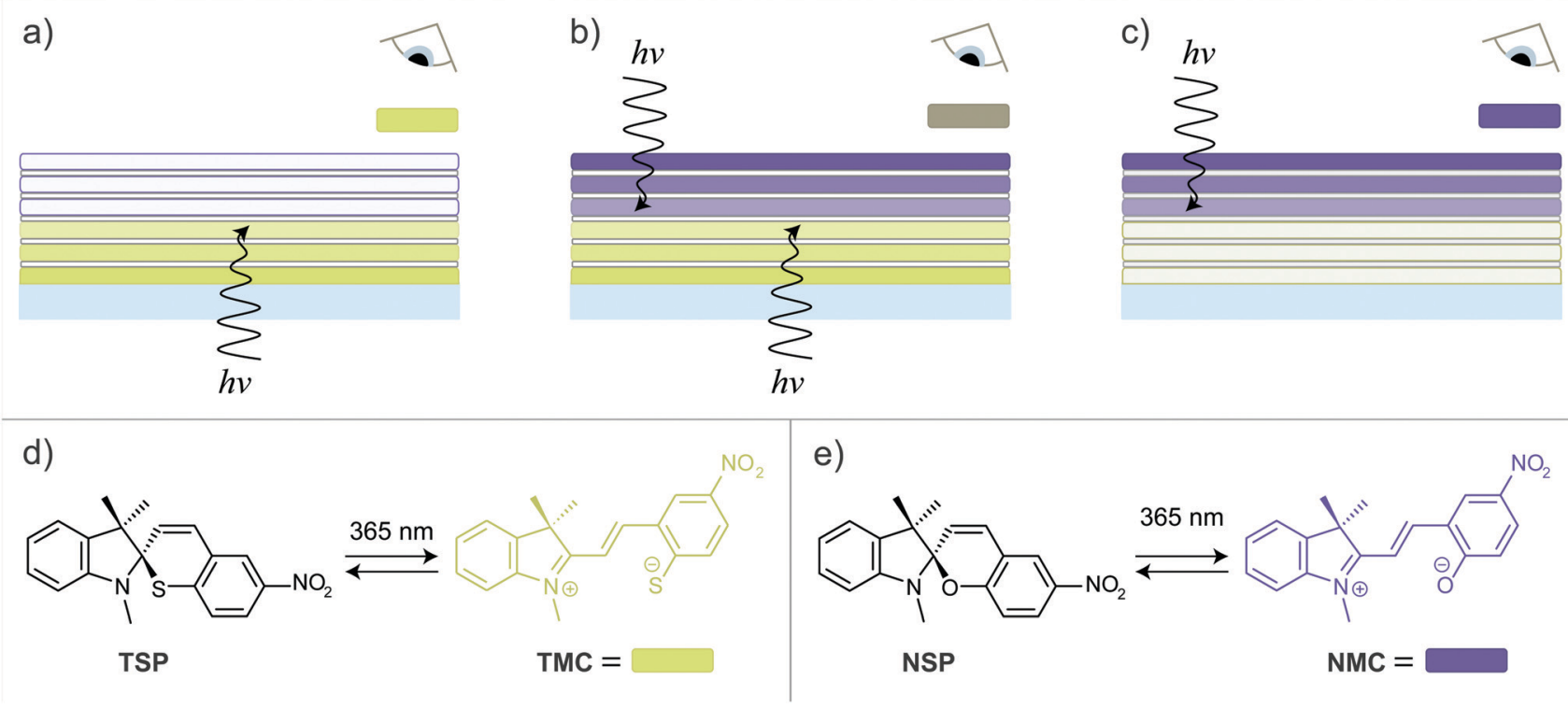

Scheme 1 Colouration obtained by selective irradiation of photochrome-containing multilayers deposited on the glass. (a) Short irradiation of one side of the filter yields green, (b) irradiation of both sides yields grey, and (c) irradiation of the other side yields purple. Ring-opening of the (d) nitrothiospiropyran (TSP) and (e) nitrospiropyran (NSP) photochromes to their merocyanine forms TMC and NMC, respectively. 
material is irradiated, as well as the duration of irradiation. In this way, a variable colour transmission filter is formed, in which the distinct absorption spectra of two spiropyran photochromes allow for a wide range of colours to be generated by spatial control of irradiation at a single wavelength. This colour filter can be adapted for use in specific applications by changing the type and concentration of photochromic switches used to tune colouration and response times, demonstrated with the use of a dithienylethene photochrome also.

\section{Experimental}

TSP $\quad((R)$-1,3,3-trimethyl-6'-nitrospiro[indoline-2,2'-thiochromene]) was prepared using methods from the literature and its characterisation is provided in the $\mathrm{ESI}^{\dagger}$ (Scheme S1). ${ }^{23}$ Solvents and reagents were obtained from Sigma-Aldrich (PMMA and HEC) or TCI and used as received unless stated otherwise. NSP $\left((R)-1,3,3\right.$-trimethyl-6' ${ }^{\prime}$-nitrospiro[indoline-2,2' chromene]) was used as received from TCI. DTE (1,2-bisterthienyl-hexafluorocyclopentene) was available from earlier studies. ${ }^{24}$

NMR spectra were recorded using a Bruker Avance 400 spectrometer. Fourier transform infrared (FTIR) spectra were recorded using a PerkinElmer Spectrum 400 FTIR spectrometer equipped with an ATR unit, or using a JASCO 4600 FTIR spectrometer in the transmission mode. UV/vis absorption spectra were recorded using an AnalytikJena Specord 600 spectrometer. Irradiation at $365 \mathrm{~nm}$ was carried out with a fibre coupled LED (Thorlabs M365F1, $4.1 \mathrm{~mW}$ ) with the output of the fibre collimated with a planoconvex lens. Film thicknesses were determined by profilometry using a Bruker Dektak XT profilometer. FTIR absorption spectroscopy was used to determine the average film thickness making use of the BeerLambert-Bougier law and the $\mathrm{CH}$ stretching bands at $c a$. $3000 \mathrm{~cm}^{-1}$ of PMMA that are at a higher wavenumber than the optical cut-off of glass ( $c a .2100 \mathrm{~cm}^{-1}$ ). A calibration curve correlated the integrated absorbance of the bands of PMMA in the range 2624 to $3182 \mathrm{~cm}^{-1}$ with the film thickness determined by profilometry (Fig. S1, ESI $\dagger$ ) from which an average film thickness over $c a$. $0.5 \mathrm{~cm}^{2}$ could be determined more readily. It is notable that the absorbance correlated well with the \%PMMA in the solutions used for spin coating also (Fig. S2, $\mathrm{ESI} \dagger)$.

PMMA films containing spiropyran (NSP or TSP) were prepared by spin coating solutions of spiropyran at various concentrations with $2-16 \mathrm{wt} \%$ of PMMA $\left(M_{\mathrm{w}} 120 \mathrm{kDa}\right)$ in solvent (DCM, toluene, and ethyl acetate) on quartz or glass microscope slides (Knittel Glass 3x1 inch microscope slides). A Laurell WS650MZ-23NPPB spincoater was used in static mode with (1) $2 \mathrm{~s}$ at $400 \mathrm{rpm}$ (acceleration $400 \mathrm{rpm} \mathrm{s}^{-1}$ ), (2) $1.5 \mathrm{~s}$ at $1500 \mathrm{rpm}$ (acceleration $1500 \mathrm{rpm} \mathrm{s}^{-1}$ ), and (3) $30 \mathrm{~s}$ at $4000 \mathrm{rpm}$ (acceleration $1200 \mathrm{rpm} \mathrm{s}^{-1}$ ) unless stated otherwise. Multi-layer coatings were prepared by alternate layering of PMMA and hydroxyethyl cellulose (HEC). HEC layers were formed by spin coating 0.25 to $0.5 \mathrm{wt} \%$ aqueous solutions of HEC on the
PMMA layers. NIR emission spectra were recorded using an iDus-InGaAs-DU490A diode array detector coupled to a Kymera193i spectrograph (ANDOR Technology). Excitation at $354.67 \mathrm{~nm}$ was provided by an Innolas Spitlight400 Nd-YAG laser (6 ns pulse width, $10 \mathrm{~Hz}, 2 \mathrm{~mJ}$ per pulse) at $90^{\circ}$ to the collection axis. The sample $(2 \mathrm{~mL})$ was held in a $1 \mathrm{~cm}$ quartz cuvette with continuous stirring during irradiation.

\section{Results and discussion}

Both NSP and TSP undergo ring opening at the spiro centre upon irradiation with UV light to yield their coloured merocyanine forms. ${ }^{25-27}$ Their photochromism in solution was studied in ethyl acetate to mimic best the solvation properties of the polymer PMMA used as the host matrix in the films discussed below. NSP and TSP absorb similarly in the UV region, and upon irradiation to the photostationary states at $365 \mathrm{~nm}$ $\left(\mathrm{PSS}_{365 \mathrm{~nm}}\right)$ they show complementary coverage of the visible absorption spectrum (Fig. 1). In both cases the absorbance at $365 \mathrm{~nm}$ is essentially unchanged by irradiation as the two isomers (SP/MC) have the same molar absorptivity at that wavelength.

\section{Preparation and characterisation of NSP and TSP containing PMMA films}

Thin films of PMMA were prepared by spin-coating ${ }^{28}$ from toluene, dichloromethane or ethyl acetate. Even though the effect of specific parameters on the film thickness is known, predicting the final thickness of a coating in a particular situation requires attention to certain parameters. Angular velocity, solution viscosity and concentration of PMMA are reported to have a dominant effect, whilst deposition rate and rotation acceleration have a modest or negligible effect on the final film properties. ${ }^{28,29}$ In the present study (after a short optimisation), all parameters were held constant, except for the concentration of PMMA, which was varied from 2 to 16 wt\% PMMA to obtain a range of film thicknesses. Film a)

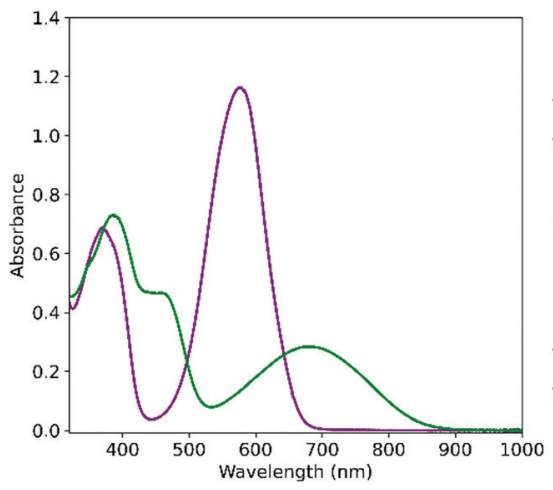

b)
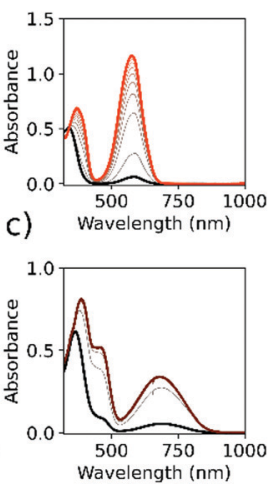

Fig. 1 (a) UV/Vis absorption spectra of NMC (purple) and TMC (green) in PMMA films on glass slides. Photochemical switching to their PSS $_{365 \mathrm{~nm}}$ of (b) NSP (black to red) and (c) TSP (black to brown) at room temperature. The films were spun from a $2 \mathrm{wt} \%$ solution of PMMA in dichloromethane containing $20 \mathrm{mM}$ NSP or TSP. 
uniformity (flatness) determined by profilometry was good for films spun from solutions with up to $8 \mathrm{wt} \%$ PMMA; however, textured films were obtained with solutions of approximately $16 \mathrm{wt} \%$ in ethyl acetate (Fig. S1, ESI $\dagger$ ).

NSP and TSP containing films were prepared by dissolving the respective photochrome in the PMMA-containing solution used for spin-coating. The photochemical switching of PMMA films loaded with either NSP or TSP shows the expected change in visible absorbance and essentially constant absorbance at the wavelength of irradiation (365 nm) (Fig. 1). Films with a final absorbance at $365 \mathrm{~nm}$ of less than 0.8 allow for the maximum conversion of the spiropyran form to the merocyanine form to be reached without limitations due to primary inner filter effects (vide infra).

Films containing NSP, which absorb essentially all UV light, show close to $0 \%$ transmission of visible light between 520 and $600 \mathrm{~nm}$ at the $\mathrm{PSS}_{365 \mathrm{~nm}}$, whereas for a comparable film containing TSP the visible transmission is only reduced to $50 \%$. The complementarity of the absorption spectra of the NMC and TMC merocyanine isomers allows for full coverage of the visible absorption spectrum. The difference in performance of the two photochromes can be understood by consideration of their respective absorption spectra of each alone in optically dilute solution at their respective PSS $_{365 \mathrm{~nm}}$ (Fig. 1). NSP and TSP differ substantially in their absorbance in the visible region at the PSS $_{365 n m}$ relative to their absorbance in the UV region. For TSP, the maximum visible absorbance of its merocyanine form at the $\mathrm{PSS}_{365 \mathrm{~nm}}$ relative to the absorbance at $365 \mathrm{~nm}$ is approximately four times lower than that of NSP. It should be noted that TMC and NMC have comparable oscillator strengths for the visible transitions and the difference is primarily due to the broadness of the absorption bands of TMC relative to NMC. Differences in the relative quantum yields for ring opening and closing reactions are likely to be of lesser importance since ring opening is much more efficient for both. The difference in the spectra of the merocyanine forms explains the limit to the optical attenuation that can be achieved in the films where the absorbance in the UV is high enough to result in a gradient of concentration of merocyanine through the depth of the films at the $\mathrm{PSS}_{365 \mathrm{~nm}}$. Ultimately the ratio in absorbance at $365 \mathrm{~nm}$ and in the visible region defines the maximum change in visible transmittance that can be achieved in films containing photochromes and is an important design aspect for applications. Since NSP and TSP are expected to disperse uniformly through the polymer film in an isotropic manner, the absorbance of each determined in solution can be used reliably for calculation of their concentration from their absorbance in the spin-coated films. Differences in refractive index (1.344 for acetonitrile vs. 1.49 for $\mathrm{PMMA}^{30}$ ) and optical interference have a minor effect on the measured absorbance and can be neglected. The relationship between the concentration of NSP in solutions of PMMA used for spin coating and the final concentration (absorbance) in the spin-coated film, as well as the effect of concentration of the photochrome in the film on the photochromic behaviour, was investigated.

The concentration of spiropyran in the solution used for spin coating correlates well with the absorbance, and therefore

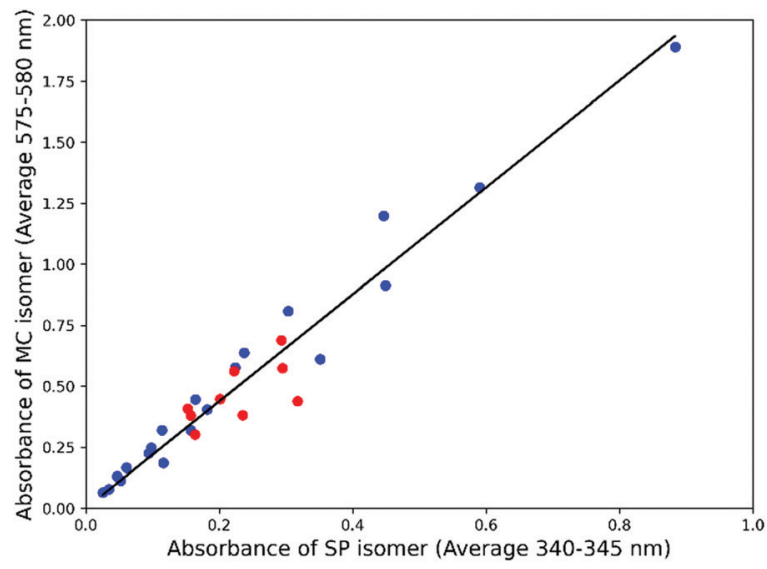

Fig. 2 Average absorbance between 575 and $585 \mathrm{~nm}$ after $10 \mathrm{~min}$ irradiation at $365 \mathrm{~nm}$ with respect to the average initial absorbance between 340 and $345 \mathrm{~nm}$ for films of PMMA of various thicknesses on the glass containing various concentrations of NSP (slope $=2.19$, $\left.R^{2}=0.95\right)$. An independent data set, prepared from new stock solutions, is shown in red.

with the concentration of spiropyran in the PMMA films (Fig. 2). Furthermore, the visible absorbance at PSS $_{365 \mathrm{~nm}}$ correlates well with the initial UV absorbance of films up to an absorbance of 1 at $365 \mathrm{~nm}$ (Fig. 2). Taken together, the concentration of spiropyran in the PMMA containing solution can be used to predict the final visible absorbance of the irradiated PMMA film.

Since PMMA can contain residual (acrylic) acid groups, the occurrence of acidochromism in the spiropyran-functionalised films should also be taken into account. The acidochromism of NSP was studied earlier ${ }^{31}$ and shows that protonation with strong acids leads to the spontaneous formation of the protonated merocyanine form $Z$-NMCH${ }^{+}$, which undergoes $E-Z$ isomerisation both thermally and photochemically. Both $Z$-NMCH${ }^{+}$and $E$-NMCH${ }^{+}$absorb only in the near blue region $(<450 \mathrm{~nm})$ of the visible spectrum. Such a shift in absorption to the blue was not observed to a significant extent and hence the expected residual acidity in PMMA is likely to be insufficient to result in protonation of the spiropyrans. Indeed, $\mathrm{HCl}$ vapours have only a modest effect on the absorption spectra of the spiropyrans in PMMA films (Fig. S3, ESI $\dagger$ ).

Although it is advantageous to use thinner films of PMMA to ensure uniformity in film thickness, high concentrations of the photochrome result in the spiropyran and merocyanine forms contributing substantially to the 'solvent properties' of the film, and in particular to an increase in polarity as the zwitterionic merocyanine form is generated. ${ }^{16}$ This effect is manifested in the rate and maximum extent of the photochromic response in isoabsorptive films, in which the final concentration of the spiropyran and the thickness of the film were varied inversely (Fig. 3). A decrease in response rate and in maximum extent of conversion to the merocyanine form was observed at the highest concentrations. Hence, a maximum rate and extent is reached with thicker films with a lower concentration of photochrome. 
a)

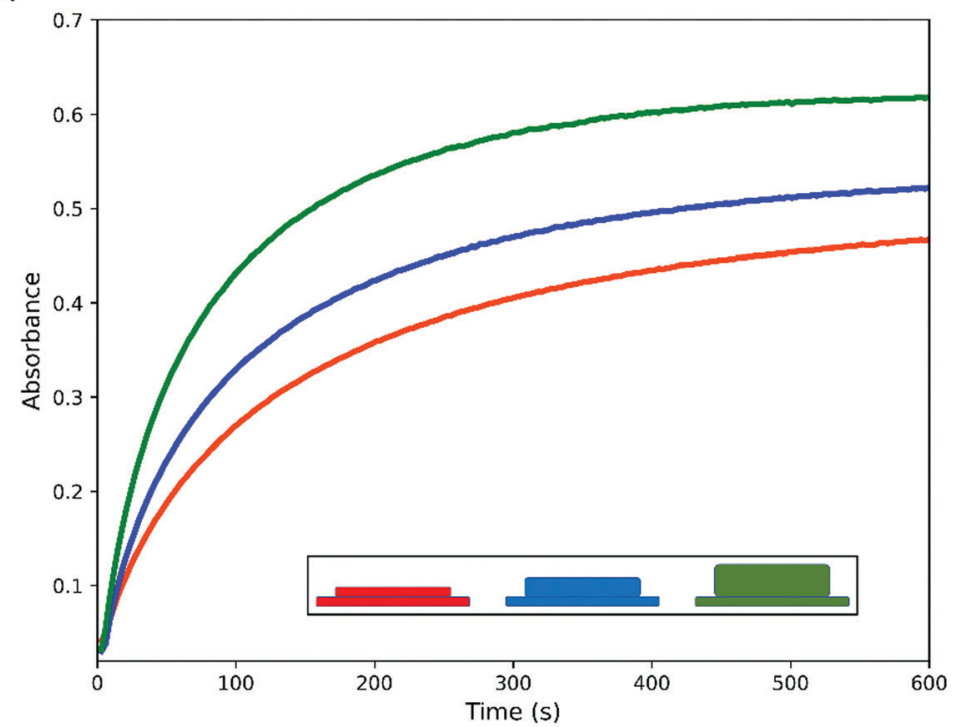

b)

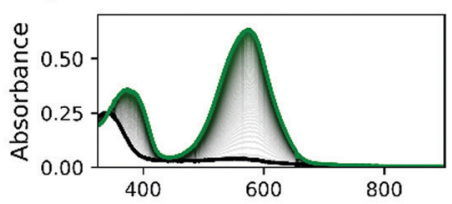

c)

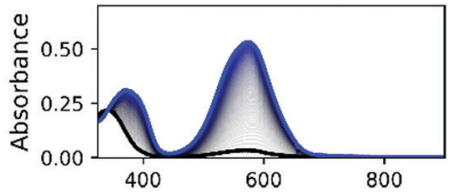

d)

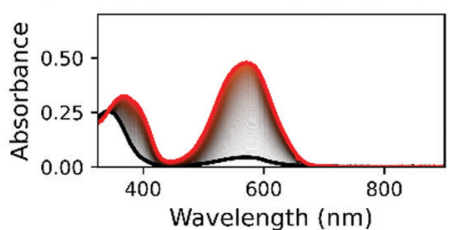

Fig. 3 (a) Trajectory towards the PSS $365 \mathrm{~nm}$ for NSP containing films of PMMA during irradiation. The initial absorbance at $365 \mathrm{~nm}$ was held the same for all three films by compensating for film thickness with a change in concentration of NSP. (b-d) UV/vis absorption spectra of each slide before (black), during (grey) and after (colour corresponds to line colour in panel (a)) irradiation.

As discussed above, thicker PMMA films can be obtained by spin-coating more concentrated solutions of PMMA but this results in a lower uniformity in film thickness (Fig. S1, ESI $\dagger$ ). As film thickness comes close to the wavelength of light used for irradiation, optical effects (e.g., interference, waveguiding) may play a role in determining the photochemical and optical behaviour of the films. Furthermore, multiple layers of PMMA need to be deposited to have spatial separation of the photochromes (NSP and TSP) in the film. An increase in the total absorbance of the films was best achieved by applying multiple layers of PMMA from solutions containing $8 \mathrm{wt} \%$ of polymer. An immediate challenge in applying sequential layers is that the solutions used for the second layer removed the first layer applied and hence a uniform build-up of film thickness could not be achieved. This interference could be circumvented by using a polar solvent, such as water, to form an interlayer of the polymer which has a substantially different solubility than PMMA. The intermediate layer should not affect the properties of the PMMA layers nor be of a thickness that leads to optical interference due to a mismatch in refractive index. Previous studies on thin films with different hydrophobicity shows that layers below the limit of detection by IR absorption spectroscopy still show differences in hydrophobicity and therefore such thin layers can show the desired effect. ${ }^{32}$ The interlayer approach allowed for multiple layers of HEC and photochromecontaining PMMA films to be built up on glass slides (Fig. 4). Hydroxyethyl cellulose (HEC), a cellulose-based water-soluble polymer, provides the desired properties and its low solubility in water (ca. $1 \mathrm{wt} \%)$ and the high viscosity of its aqueous solutions allows for thin films to be formed that are sufficiently a)

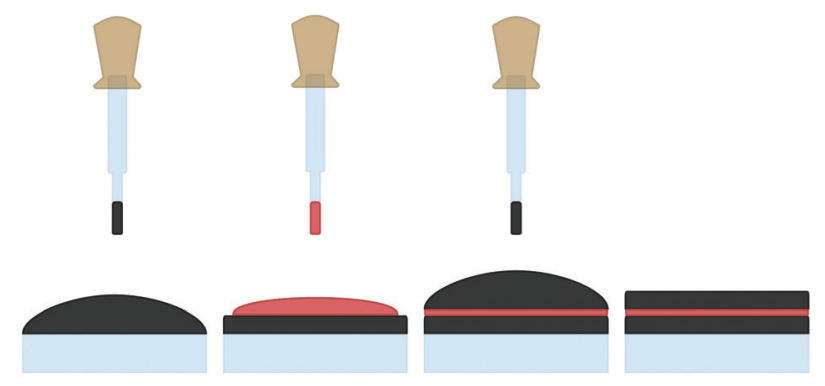

b)

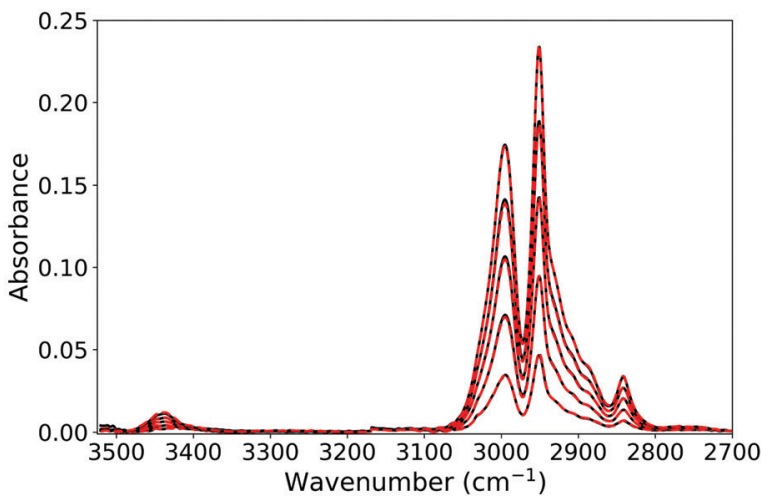

Fig. 4 (a) Deposition of multiple layers by alternate spin-coating of PMMA and HEC containing solutions and (b) FTIR absorption spectra of a glass slide coated alternately with 10 layers of PMMA (8 wt\%) from ethyl acetate and HEC (0.25 wt\%) from water. The spectra after deposition of each PMMA layer are shown in black and of each HEC layer in red. The $\mathrm{O}-\mathrm{H}$ stretching band of $\mathrm{HEC}$ at $3400 \mathrm{~cm}^{-1}$ and $\mathrm{C}-\mathrm{H}$ stretching bands of both $\mathrm{HEC}$ and PMMA at $2800-3000 \mathrm{~cm}^{-1}$ are observed in this region. 
hydrophilic to prevent dissolution of the underlying PMMA layer during spin coating of subsequent PMMA containing solutions.

\section{Tuneable visible transmission filters based on multi-layered TSP/NSP coatings}

Layers of PMMA containing TSP and NSP were applied with the final UV absorbance of the films being a combination of the absorbance of each constituent layer (Scheme 1). Irradiation at $365 \mathrm{~nm}$ from either side of the film induces ring opening to the merocyanine form and a decrease in transmittance across the visible spectrum, which is a weighted sum of the spectrum of the respective merocyanine isomers TMC and NMC (Fig. 5 and Fig S4, ESI $\dagger$ ). The spectrum obtained depends on the order with which the photochrome containing layers are deposited in the multi-layered material and on the side of the film that is irradiated at $365 \mathrm{~nm}$ due to the primary inner filter effect. When the number of layers of PMMA containing each photochrome was sufficient to absorb all light at $365 \mathrm{~nm}$ (in this case three layers of each were used with HEC interlayers), photochemical switching of the other photochrome in the layers beneath was prevented (Scheme 1(a) and (c)). Indeed, irradiation from the NSP coated side results in maximum switching of NSP to NMC with the TSP in the layers behind unchanged, and similarly, irradiation from the TSP side (through the glass support) results in maximum conversion of TSP to TMC without significant conversion of NSP. The dependence on the direction of irradiation allows for the extent of switching of NSP and TSP to be controlled independently and therefore the final transmission spectrum of the film to be tuned. For slides composed of multilayers of PMMA, in which an equal amount of NSP and TSP is present in each layer (simple mixture), the changes in UV/vis transmission are independent of the side from which irradiation is carried out (Fig. S5, ESI $\dagger$ ).

The approach taken to multi-layered films is general to other classes of photochromic molecular switches, such as dithienylethene (DTE) photochromes, ${ }^{24}$ for single wavelength colour tuning filters. Separately switching SP or DTE with the same wavelength of irradiation is achieved as before by choosing the side of irradiation yielding differently coloured films (Fig. 6 and Fig. S6, ESI $\dagger$ ). The advantage of using DTE switches is their robustness and thermal stability, compared with, for example, TSP, but they show a lower quantum yield for conversion from coloured to colourless form. Using different switch classes and being able to use different thin layers with different spin coating solvents or even polymers and polymer concentrations creates a wide range of possibilities to tune the photochromic filter to the desired application.

Some deviations were found in the various samples prepared, especially when spin-coating the intermediate HEC layers. The high surface tension of water on a hydrophobic PMMA support can lead to areas that do not wet when the aqueous HEC solution is applied in insufficient volumes. This effect is not immediately observable. It becomes apparent upon irradiation at $365 \mathrm{~nm}$ manifested in differently coloured areas depending on whether or not the HEC solution could wet. Where it could not wet (and hence form the interlayer) mixing of the photochromes between layers during deposition occurred (Fig. 7). Spreading the HEC containing solution over the slide prior to spinning provided for full coverage (Fig. 4).

The stability of TMC upon irradiation is low relative to that of NMC both in solution and in PMMA films. This is a)
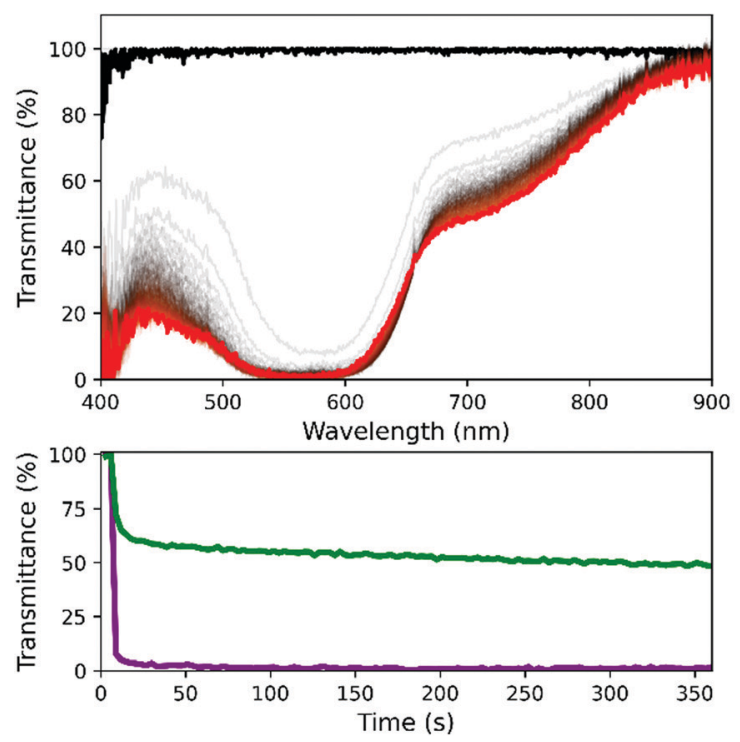

b)
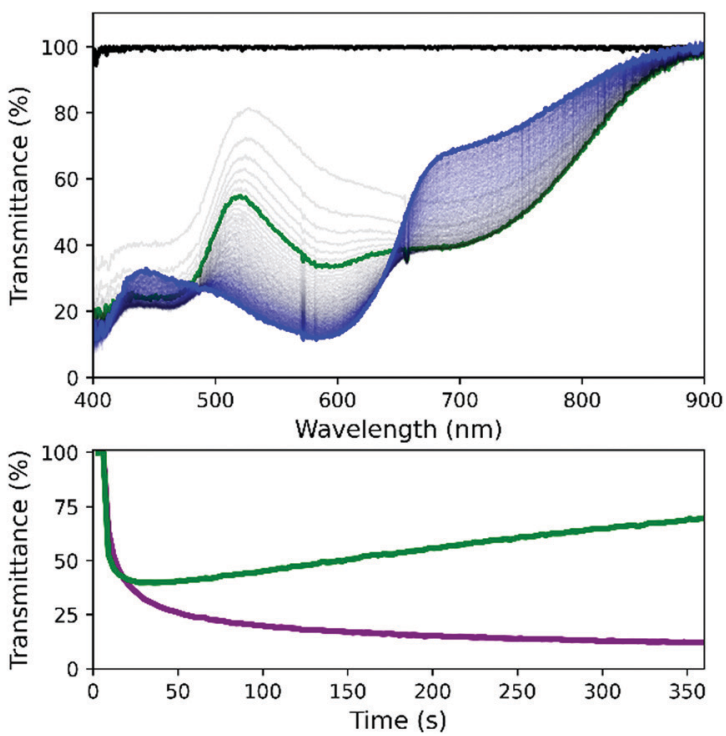

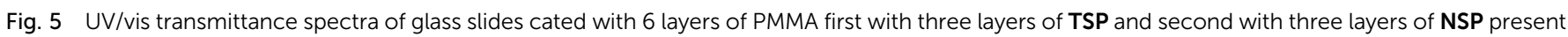

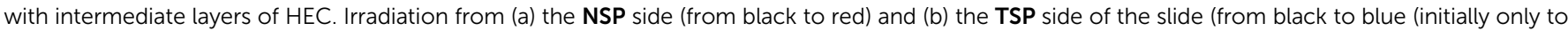

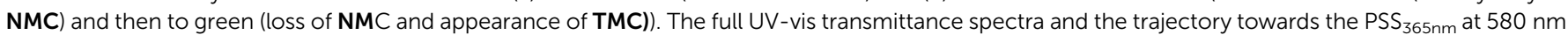
(purple) and $700 \mathrm{~nm}$ (green) are shown in each case. 
<smiles>[R]c1cc(C2=C(c3cc([R])sc3C)CCC2)c(C)s1</smiles>

DTE - colourless form<smiles>[R]c1ccc(-c2cccs2)s1</smiles>

a)

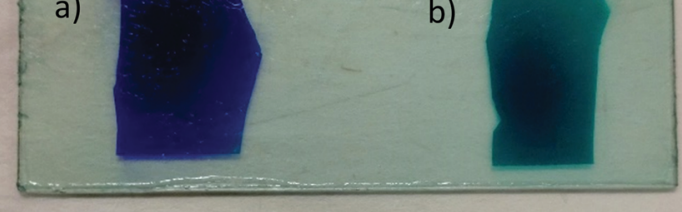

Fig. 6 Structure of DTE (12-bis-terthienyl-hexafluorocyclopentene) and glass slides coated with several layers of PMMA containing first NSP and then DTE. Irradiation at $365 \mathrm{~nm}$ on the side with (a) NSP and (b) DTE provides for differently coloured slides.

manifested in imperfect performance of the multilayer NSP/TSP films in which initially only TMC is formed when irradiation is from the side of the film containing it. Over time, the absorption spectrum changes to that of NMC, which can be understood by the loss in absorbance of TSP/TMC at $365 \mathrm{~nm}$ as it photobleaches, and hence the protective inner filter effect is lost. Therefore, although the TMC in the PMMA films appears stable to photobleaching initially, this is due to only the outermost layer undergoing conversion to the merocyanine form and as the TMC in these upper layers degrades, TSP present in deeper layers can then undergo switching until eventually the absorbance of all layers containing TSP falls to zero at $365 \mathrm{~nm}$ (Scheme 2 and Fig. 5). Degradation of TSP can be ascribed to degradation by reaction with ${ }^{1} \mathrm{O}_{2}$ generated under irradiation of a)

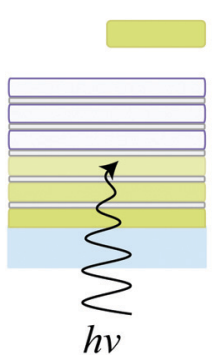

b)

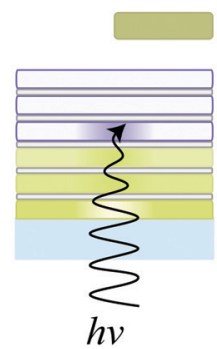

c)

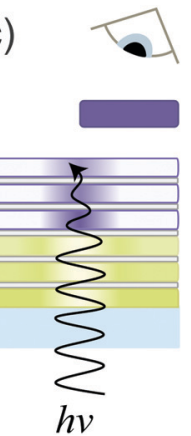

Scheme 2 Photobleaching upon continuous irradiation of the side of the slide with PMMA containing TSP is manifested in a colour change over time, as the TMC is degraded allowing for the NSP to switch to the NMC form (a to $\mathrm{c}$ )

$365 \mathrm{~nm}$ as seen for DTE polymer films earlier. ${ }^{24}$ Direct observation of ${ }^{1} \mathrm{O}_{2}$ produced from TSP in solution was made with pulsed excitation at $355 \mathrm{~nm}$ (Fig. S8, ESI $\dagger$ ), showing that ${ }^{1} \mathrm{O}_{2}$ also reacts with TMC efficiently.

\section{Conclusion}

We have successfully demonstrated a single wavelength switchable photochromic optical filter making use of stacked polymer films containing spiropyrans. The resulting multi-layered filter is able to attain an array of spectral densities by varying the side of incident light, and duration and intensity of illumination. The thickness of single layers of TSP and NSP containing PMMA films was kept below one micron to ensure reproducible film topology. Intermediate layers of HEC enable stacking of films to increase the optical density. The final filter comprised of stacked NSP and TSP containing layers could attain purple and yellow, or mixtures thereof, by illumination from the NSP and TSP sides. This enables the use of a single filter for applications that need variation in optical densities in different ranges of the spectrum with a single excitation wavelength, and hence simplified device construction. Furthermore, for systems in which illumination from the front is not feasible,
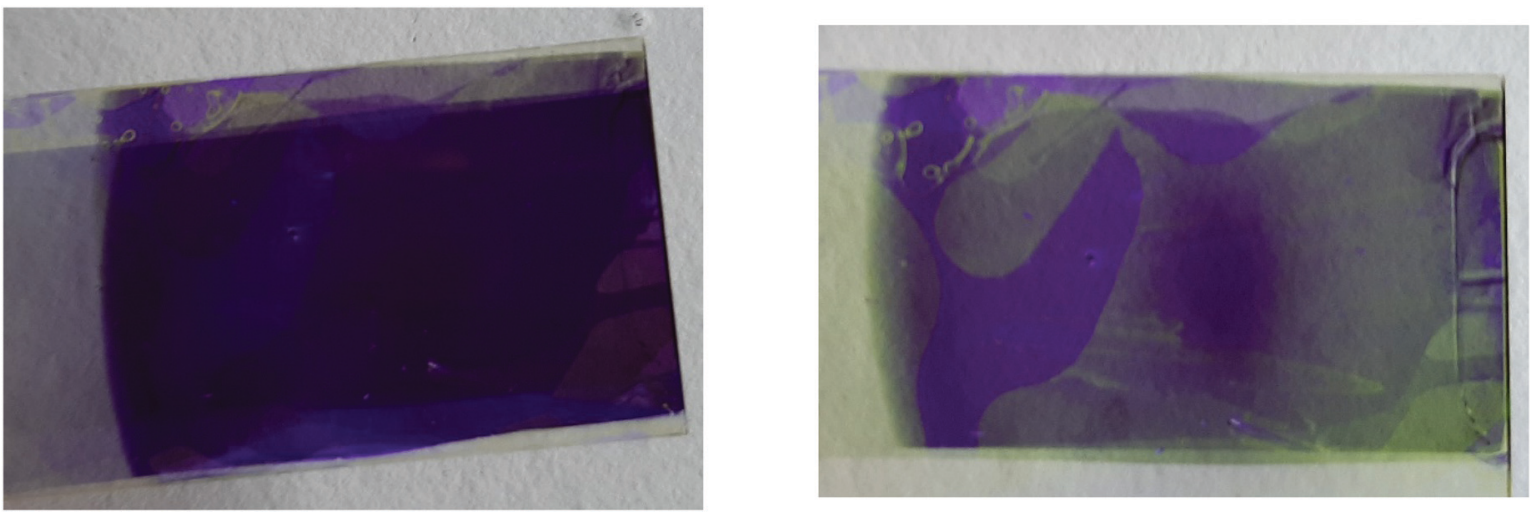

Fig. 7 Glass slides coated with several layers of PMMA with first TSP and then NSP after irradiation at $365 \mathrm{~nm}$ on the side with (left) NSP and (right) TSP. In addition to showing the generally protective inner filter effect, the patterning shows how an insufficient volume of HEC containing solution for the spin coating of intermediate layers leads to imperfect coverage and allows for sublayers of PMMA to be removed by subsequent washing steps (Fig. S7, ESI $\dagger$ ). 
illumination by wave-guiding of light along the glass support can be used for side on illumination of a coated glass. Since the inner filter effect protects the outer layer from switching, uniformly switched films in an on/off sense can be formed even where uniformity of illumination is not readily achieved. Future work will be directed towards implementation of this method using different switches to obtain other colours in the filter and increase the robustness of the photochemical performance.

\section{Conflicts of interest}

There are no conflicts to declare.

\section{Acknowledgements}

Financial support from the Netherlands Ministry of Education, Culture and Science (Gravity Program 024.001.035, WRB) and the Netherlands Organization for Scientific Research (NWO) through the Advanced Research Centre Chemical Building Blocks Consortium (2018.015.C RUG) is acknowledged.

\section{References}

1 H. Ghiradella, D. Aneshansley, T. Eisner, R. E. Silberglied and H. E. Hinton, Science, 1972, 178, 1214-1217.

2 H. E. Hinton and G. M. Jarman, J. Insect Physiol., 1973, 19, 533-549.

3 K. Nassau, Color Res. Appl., 1987, 12, 4-26.

4 J. Teyssier, S. V. Saenko, D. van der Marel and M. C. Milinkovitch, Nat. Commun., 2015, 6, 6368.

5 F. Lang, H. Wang, S. Zhang, J. Liu and H. Yan, Int. J. Thermophys., 2018, 39, 6.

6 O. Melnyk, R. Jones, R. Macêdo, Y. Garbovskiy, G. Hagen, A. V. Glushchenko, K. Spendier and R. E. Camley, ACS Photonics, 2021, 8, 1222-1231.

7 S. Sahli, J. Hayward, Q. Fang and R. M. Y. M. Abdlaty, Proc. SPIE, 2018, 10573, 105732P.

8 E. S. Wachman, W. Niu and D. L. Farkas, Biophys. J., 1997, 73, 1215-1222.

9 J. Vila-Francés, J. Calpe-Maravilla, J. Muñoz-Mari, L. GómezChova, J. Amorós-López, E. Ribes-Gómez and V. DuránBosch, Rev. Sci. Instrum., 2006, 77, 073108.

10 W. R. Browne and B. L. Feringa, Annu. Rev. Phys. Chem., 2009, 60, 407-428.
11 K. Matsuda and M. Irie, J. Photochem. Photobiol., C, 2004, 5, 169-182.

12 Y. Wakayama, R. Hayakawa and H.-S. Seo, Sci. Technol. Adv. Mater., 2014, 15, 024202.

13 A. Fihey, A. Perrier, W. R. Browne and D. Jacquemin, Chem. Soc. Rev., 2015, 44, 3719-3759.

14 H. Dürr and H. Bouas-Laurent, Photochromism, Elsevier, 2003.

15 L. Wang, Y. Liu, X. Zhan, D. Luo and X. Sun, J. Mater. Chem. C, 2019, 7, 8649-8654.

16 A. Abdollahi, Z. Alinejad and A. R. Mahdavian, J. Mater. Chem. C, 2017, 5, 6588-6600.

17 V. A. Barachevsky, A. O. Ait, A. M. Gorelik, T. M. Valova, N. L. Zaichenko, L. S. Kol'tsova, A. I. Shienok, V. P. Grachev and S. M. Aldoshin, Russ. J. Gen. Chem., 2018, 88, 2773-2786.

18 B. L. Feringa and W. R. Browne, Molecular Switches, WileyVCH, Weinheim, 2011.

19 M. M. Lerch, M. J. Hansen, W. A. Velema, W. Szymanski and B. L. Feringa, Nat. Commun., 2016, 7, 1-10.

20 T. Stauch and A. Dreuw, J. Phys. Chem. Lett., 2016, 7, 1298-1302.

21 R. Klajn, Chem. Soc. Rev., 2014, 43, 148-184.

22 T. Komikado, A. Inoue, K. Masuda, T. Ando and S. Umegaki, Thin Solid Films, 2007, 515, 3887-3892.

23 Y. Shiraishi, K. Tanaka, E. Shirakawa, Y. Sugano, S. Ichikawa, S. Tanaka and T. Hirai, Angew. Chem., Int. Ed., 2013, 52, 8304-8308.

24 L. Kortekaas and W. R. Browne, Adv. Opt. Mater., 2016, 4, 1378-1384.

25 E. Fischer and Y. Hirshberg, J. Chem. Soc., 1952, 4522-4524.

26 R. S. Becker and J. Kolc, J. Phys. Chem., 1968, 72, 997-1001.

27 S. Arakawa, H. Kondo and J. Seto, Chem. Lett., 1985, 1805-1808.

28 K. Norrman, A. Ghanbari-Siahkali and N. B. Larsen, Annu. Rep. Prog. Chem., Sect. C: Phys. Chem., 2005, 101, 174-201.

29 A. G. Emslie, F. T. Bonner and L. G. Peck, J. Appl. Phys., 1958, 29, 858-862.

30 N. Sultanova, S. Kasarova and I. Nikolov, Acta Phys. Pol., A, 2009, 116, 585-587.

31 L. Kortekaas, J. Chen, D. Jacquemin and W. R. Browne, J. Phys. Chem. B, 2018, 122, 6423-6430.

32 H. Logtenberg, M. J. Lopez-Martinez, B. L. Feringa, W. R. Browne and E. Verpoorte, Lab-on-a-Chip, 2011, 11, 2030-2034. 